\title{
Near-Infrared Spectral Energy Distributions of Seyfert Galaxies: Stellar Population, Active Nucleus, and Hot Dust
}

\author{
Rogério Riffel $^{1}$, Miriani G. Pastoriza ${ }^{1}$, Alex Carciofi ${ }^{2}$, \\ Alberto Rodríguez-Ardila ${ }^{3}$, and Charles Bonatto ${ }^{1}$ \\ ${ }^{1}$ Departamento de Astronomia, Universidade Federal do Rio Grande do Sul. Av. Bento \\ Gonçalves 9500, Porto Alegre, RS, Brazil \\ Email: riffel@ufrgs.br \\ ${ }^{2}$ Departamento de Astronomia do IAG/USP, Rua do Matão 1226, São Paulo, SP, Brazil \\ ${ }^{3} \mathrm{LNA} / \mathrm{MCT}$ - Rua dos Estados Unidos 154, Itajubá, MG, Brazil
}

Keywords. galaxies: active, galaxies: stellar content, stars: AGB and post-AGB.

We investigate the NIR spectra of 24 Seyfert galaxies observed with the instrument SpeX at the NASA Infrared Telescope Facility (ITRF) in the short cross-dispersed mode. The results of the spectral synthesis fitting procedure are presented and discussed in details by Riffel et al. (2009). The approach followed here is based on the STARLight code (see Cid Fernandes et al. 2005). The spectral synthesis shows that the NIR continuum of active galaxies can be explained in terms of at least three components: a non-thermal continuum, dust emission, and the stellar population of the circumnuclear region. The study of the stellar population is a critical step in the analysis of the continuum emission of Seyfert galaxies. Moreover, our results are consistent with the predictions of the unified model for AGNs, as the non-thermal continuum and the hot dust emission are present in all Sy 1 sources and only in a small fraction of the Sy 2s. Regarding the stellar population component, our results point to a mean metallicity solar to above solar, if we consider the light-weighted values, while for the mass-weighted mean metallicity our results indicate a sub-solar value. We associate this discrepancy with the well known age-metallicity degeneracy: i.e., for a fixed mass, a high-metallicity stellar population looks cooler - and older - than a low-metallicity population, thus resulting in a higher $M / L$ ratio. Moreover, this is consistent with a galaxy chemical enrichment scenario in which the young population is enriched by the evolution of the early massive stars. In this context, the light-weighted metallicity is more sensitive to the young component, while the mass-weighted metallicity to the old stellar population. The main results can be summarized as follows:

(i) The NIR stellar population synthesis does not reproduce the optical results well.

(ii) Our synthesis shows significant differences between Sy 1 and Sy 2 galaxies. The hot dust component is required to fit the $K$-band spectra of $\sim 80 \%$ of the Sy 1 galaxies, and only of $\sim 40 \%$ of the Sy $2 \mathrm{~s}$. Besides, about $50 \%$ of the Sy 2 galaxies require a featureless continuum contribution in excess of $20 \%$, while this fraction increases to about $60 \%$ in the Sy $1 \mathrm{~s}$. Also, in about $50 \%$ of the Sy $2 \mathrm{~s}$, the combined featureless continuum and young stellar population components contribute with more than $20 \%$, while this occurs in $90 \%$ of the Sy 1 . This suggests recent star formation in the central region of our galaxy sample.

(iii) The light at $1.223 \mu \mathrm{m}$ in the central regions of the galaxies contains a substantial fraction from intermediate-age stellar populations with a mean metallicity near solar. Our analysis confirms that the $1.1 \mu \mathrm{m} \mathrm{CN}$ band can be used as an unambiguous tracer of intermediate-age stellar populations.

\section{References}

Cid Fernandes, et al. 2005, MNRAS, 358, 363

Riffel, R., Pastoriza, M. G., Rodríguez-Ardila, A., \& Bonatto, C. 2009 [arXiv:0907.4144] 